\title{
Exact solution of Calogero model with competing long-range interactions
}

\author{
B. Basu-Mallick ${ }^{\dagger}$ and Anjan Kundu * \\ Saha Institute of Nuclear Physics, Theory Group \\ 1/AF Bidhan Nagar, Calcutta 700 064, India.
}

\begin{abstract}
An integrable extension of the Calogero model is proposed to study the competing effect of momentum dependent long-range interaction over the original $\frac{1}{r^{2}}$ interaction. The eigenvalue problem is exactly solved and the consequences on the generalized exclusion statistics, which appears to differ from the exchange statistics, are analyzed. Family of 'dual' models with different coupling constants is shown to exist with same exclusion statistics.

03.65.-w, 05.30.Pr, 05.45.-a
\end{abstract}

Particles with generalized exclusion statistics (GES) introduced by Haldane [1] is believed to play important role in 1D non-Fermi liquids as well as in the edge excitations in fractional quantum Hall effect [2, 3]. Such exclusion statistics can be realized microscopically in models like Calogero model [4] with $\frac{1}{r^{2}}$ interaction:

$$
H_{c s}=-\frac{1}{2} \sum_{i=1}^{N} \frac{\partial^{2}}{\partial x_{i}^{2}}+\frac{\omega^{2}}{2} \sum_{i=1}^{N} x_{i}^{2}+\frac{g}{2} \sum_{i \neq j} \frac{1}{\left(x_{i}-x_{j}\right)^{2}} .
$$

More precisely, the GES parameter $\nu$ can be linked with the coupling constant as [5 7 ]

$$
\nu=\frac{1}{2}(1 \pm \sqrt{1+4 g}) .
$$

On the other hand, two dimensional anyonic model in the lowest Landau level under strong magnetic field can also be related to the exclusion statistics [8]. However one observes that the GES parameter in such anyonic model is given by the coupling constant of a momentum dependent long-range interaction. Similar momentum dependent but short-range interactions were found to appear also in integrable models, like derivative $\delta$ [9] and double- $\delta$ function bose gases exhibiting again the exclusion statistics 10,11]. This motivates us to study the competing effect of a momentum dependent Coulomb-like interaction: $H_{p}=\delta \sum_{i=1}^{N} f_{i} \frac{\partial}{\partial x_{i}}$, where $f_{i}=\sum_{k \neq i} \frac{1}{x_{i}-x_{k}}$, over the $\frac{1}{r^{2}}$ interaction of Calogero model, by adding $H_{p}$ directly to Hamiltonian (11). In general such additional interaction with an independent coupling constant spoils the integrability of the original system. However, we find that the proposed model

$$
H=H_{c s}+H_{p}=-\frac{1}{2} \sum_{i=1}^{N} \frac{\partial^{2}}{\partial x_{i}^{2}}+\frac{\omega^{2}}{2} \sum_{i=1}^{N} x_{i}^{2}+\frac{g}{2} \sum_{i \neq j} \frac{1}{\left(x_{i}-x_{j}\right)^{2}}+\delta \sum_{i=1}^{N} f_{i} \frac{\partial}{\partial x_{i}},
$$

not only retains its exact solvability but also allows explicit construction of Lax pair and infinite set of conserved quantities. Moreover, the additional coupling constant $\delta$ competes now with the coupling constant $g$ to modify considerably the GES picture. In fact, as will be shown below, the GES parameter becomes functionally dependent on both the coupling constants and remains nontrivial even in the absence of the original $\frac{1}{r^{2}}$ interaction. This would give rise to a family of models with same exclusion statistics but with different sets of coupling constants lying on a parabolic curve. Furthermore, contrary to the Calogero model, the exchange statistics in the present model seems to differ from its exclusion statistics.

\footnotetext{
*email: anjan@tnp.saha.ernet.in

†biru@tnp.saha.ernet.in
} 
To reveal the above features we recall that, Calogero like models have been solved recently by mapping them to a system of free oscillators 12 15. For solving model (3) through a similar technique, we conjecture first that its ground state is given by the Laughlin-type wave function

$$
\psi_{g r}=\prod_{i<j}\left(x_{i}-x_{j}\right)^{\nu} e^{-\frac{\omega}{2} \sum_{i=1}^{N} x_{i}^{2}},
$$

$\nu$ being an unknown parameter to be determined later. Using expression (4) we may simplify the Hamiltonian (3) as

$$
H_{1}=\psi_{g r}^{-1}\left(H-E_{g r}\right) \psi_{g r}=-\frac{1}{2} \sum_{i=1}^{N} \frac{\partial^{2}}{\partial x_{i}^{2}}-(\nu-\delta) \sum_{i=1}^{N} f_{i} \frac{\partial}{\partial x_{i}}+\omega \sum_{i=1}^{N} x_{i} \frac{\partial}{\partial x_{i}},
$$

where

$$
E_{g r}=\frac{N \omega}{2}[1+(N-1)(\nu-\delta)] .
$$

For this simplification we have dropped the $\frac{1}{r^{2}}$ term by equating its coefficient to zero, which gives $g=\nu^{2}-\nu(1+2 \delta)$ and in turn determines the power index $\nu$ as

$$
\nu=\frac{1}{2}\left((1+2 \delta) \pm \sqrt{(1+2 \delta)^{2}+4 g}\right) .
$$

We notice that the parameter $\nu$, which is linked to the symmetry of the wave function (1) and related to the exchange statistics, depends on both the coupling constants of model (3) in an involved way.

We observe further that the transformed Hamiltonian (5) may be expressed as $H_{1}=S^{-}+\omega S^{3}$, with the Lassalle operator $S^{-}=-\frac{1}{2} \sum_{i=1}^{N} \frac{\partial^{2}}{\partial x_{i}^{2}}-(\nu-\delta) \sum_{i=1}^{N} f_{i} \frac{\partial}{\partial x_{i}}$ and the Euler operator $S^{3}=\sum_{i=1}^{N} x_{i} \frac{\partial}{\partial x_{i}}$, satisfying the commutation relation $\left[S^{-}, S^{3}\right]=2 S^{-}[15]$. Using therefore the well known Baker-Hausdorff transformation we can remove the $S^{-}$part from $H_{1}$ and through some additional similarity transformations reduce it finally to a free oscillator model

$$
H_{2}=\mathcal{S}^{-1} H_{1} \mathcal{S}=-\frac{1}{2} \sum_{i=1}^{N} \frac{\partial^{2}}{\partial x_{i}^{2}}+\frac{\omega^{2}}{2} \sum_{i=1}^{N} x_{i}^{2}-\frac{\omega N}{2},
$$

where $\mathcal{S}=e^{\frac{1}{2 \omega} S^{-}} e^{\frac{1}{4 \omega} \nabla^{2}} e^{\frac{\omega}{2} \sum_{i=1}^{N} x_{i}^{2}}$ and $\nabla^{2}=\sum_{i=1}^{N} \frac{\partial^{2}}{\partial x_{i}^{2}}$. This mapping allows us to write the exact eigenfunctions for the extended Calogero model (3) as

$$
\psi_{n_{1} n_{2} \cdots n_{N}}=\psi_{g r} \mathcal{S} \psi_{n_{1} n_{2} \cdots n_{N}}^{0} .
$$

Here the excitation numbers $n_{i}$ 's are positive integers obeying bosonic selection rule $n_{i} \leq n_{i+1}$ and $\psi_{n_{1} n_{2} \cdots n_{N}}^{0}$ corresponds to the symmetrized form of the free oscillator eigenfunction, which in turn is given through the product of Hermite polynomials. The symmetrization of the wave function with respect to particle coordinates, as was implemented in Calogero model [12], is also needed here to avoid singularity in the normalization of the wave function (9). Therefore, in spite of the fact that the interacting Hamiltonian (3) is convertible to the free oscillator model, the need for symmetrization shows that the many-particle correlation is in fact inherent in this model.

The eigenvalues of the Hamiltonian (3) corresponding to the states (9) will naturally be given by

$$
E_{n_{1}, n_{2}, \cdots, n_{N}}=E_{g r}+\omega \sum_{i=1}^{N} n_{i}=\frac{N \omega}{2}[1+(N-1)(\nu-\delta)]+\omega \sum_{i=1}^{N} n_{i} .
$$

It is evident that for all $n_{i}=0$, the energy $E_{n_{1}, n_{2}, \cdots, n_{N}}$ attains its minimum value $E_{g r}$. At the same time, as can be easily worked out from Eqn. (9), the corresponding eigenfunction reduces to $\psi_{g r}$ (四). This proves that $\psi_{g r}$ is indeed the ground state wave function for Hamiltonian (3) with eigenvalue $E_{g r}$. For exploring the exclusion statistics of this model, we observe that its energy spectrum (10) can be expressed exactly in the form of free oscillator: 
$E_{n_{1}, n_{2}, \cdots, n_{N}}=\frac{N \omega}{2}+\omega \sum_{i=1}^{N} \bar{n}_{i}$, where $\bar{n}_{i}=n_{i}+(\nu-\delta)(i-1)$ are quasi-excitation numbers. However such numbers are no longer integers and satisfy a modified selection rule

$$
\bar{n}_{i+1}-\bar{n}_{i} \geq(\nu-\delta)
$$

which clearly restricts the difference between the quasi-excitation numbers to be at least $\tilde{\nu}=\nu-\delta$ apart. As a consequence the extended Calogero model (3) exhibits GES property with parameter

$$
\tilde{\nu} \equiv \tilde{\nu}(\delta, g)=\frac{1}{2}\left(1 \pm \sqrt{(1+2 \delta)^{2}+4 g}\right)
$$

which is clearly a function of both the coupling constants of the model. It is obvious that for $\delta \neq 0$, the GES parameter $\tilde{\nu}$ is different from the power index $\nu$ (耳), which is responsible for the symmetry of the wave function. Therefore one may conclude that unlike Calogero model, the exclusion statistics for model (3) differs from its exchange statistics. This might lead to unusual situations, where the particles with bosonic exclusion statistics (with $\tilde{\nu}=0$ ) might have nonsymmetric wave function (with $\delta=\nu \neq 0$ ) and reversely, symmetric wave function (with $\nu=0$ ) might correspond to fermionic particles (with $\tilde{\nu}=1=-\delta$ ) or those with fractional exclusion statistics ( with $-\delta=\tilde{\nu} \neq 0$ ).

It is evident that equation (12) for fixed values of $\tilde{\nu}$ describes a family of parabolic curves in the coupling constant plane $(\delta, g)$. As a consequence of this, the competing effect of the independent coupling constants $g$ and $\delta$ can make the GES feature of (3) much richer in comparison with the Calogero model. For example, while bosonic (fermionic) excitations in the Calogero model occur only in the absence of long-range interaction, the quasi-excitations in (3) can behave as pure bosons (fermions) even in the presence of both the long-range interactions satisfying the constraint $\tilde{\nu}(\delta, g)=0 \quad(\tilde{\nu}(\delta, g)=1)$. Both of these constraints lead to the same parabolic curve $g=-\delta(1+\delta)$. A family of such parabolas with shifted apex points are generated for other values of $\tilde{\nu}$ and the lowest apex point is attained at $\tilde{\nu}=\frac{1}{2}$, where the quasi-excitations would behave as semions. Note that all points along any of these parabolic curves would correspond to different Hamiltonians having different sets of coupling constants. However all such models will have the same GES parameter $\tilde{\nu}$ and hence generate the same energy spectrum. Therefore such models may be considered as 'dual' to each other. For example, the model

$$
H_{\delta}=-\frac{1}{2} \sum_{i=1}^{N} \frac{\partial^{2}}{\partial x_{i}^{2}}+\frac{\omega^{2}}{2} \sum_{i=1}^{N} x_{i}^{2}+\delta \sum_{i=1}^{N} f_{i} \frac{\partial}{\partial x_{i}},
$$

will be dual to the Calogero model (11) with coupling constant $g=\delta(\delta+1)$.

As is well known, in Calogero model the same coupling constant $g$ may correspond to two distinct GES parameters related by a symmetry: $\nu \rightarrow 1-\nu$. To reveal such a reflection symmetry in the present case together with some other details, we may look into the projection of the surface described by equation (12) on the $(\delta, \tilde{\nu})$ plane for fixed values of $g$. This gives a family of two-branched hyperbolas, which reduce to their asymptotes at $g=0$. When $g$ changes from positive to negative values, the orientation of the hyperbolas flips by an angle $\frac{\pi}{2}$. A remarkable point is that, while in the Calogero model the minimum allowed value of the coupling constant $g$ is $-\frac{1}{4}$, the corresponding value of $g$ in the present model is $-\frac{1}{4}(1+2 \delta)^{2}$, which has no lower bound. Note further that to avoid singularity in the wave function (9) the power index $\nu$ should be positive and at the same time for physical reasons the GES parameter $\tilde{\nu}$ should not be negative. Consequently, for $g>0$ only the upper-branch hyperbolas, while for $g<0$ a segment of the right-branch hyperbolas survive (as shown in Fig. 1). As is evident from the figure, along any allowed segment with fixed $g<0$, there exists certain domain where $\tilde{\nu}$ becomes a double-valued function of $\delta$. From Eqn. (12) we can explicitly calculate this domain as $\sqrt{-g}-0.5 \leq \delta \leq \sqrt{0.25-g}-0.5$. Therefore the same set of coupling constants $g$ and $\delta$ may lead to two different values of the GES parameters revealing the reflection symmetry: $\tilde{\nu} \rightarrow 1-\tilde{\nu}$ in model (3) in a more involved way. It may also be seen from the figure that on any hyperbolic curve with fixed $g>0$, two different values of $\delta: \delta_{ \pm}=-\frac{1}{2} \pm x$, give the same $\tilde{\nu}$ for any $x$. As a consequence the models with the coupling constants $\left(g, \delta_{-}\right)$and $\left(g, \delta_{+}\right)$become dual to each other for any positive $g$. In particular, the Calogero model (1) would be dual to 


$$
H_{c s}^{(d u a l)}=-\frac{1}{2} \sum_{i=1}^{N} \frac{\partial^{2}}{\partial x_{i}^{2}}+\frac{\omega^{2}}{2} \sum_{i=1}^{N} x_{i}^{2}+\frac{g}{2} \sum_{i \neq j} \frac{1}{\left(x_{i}-x_{j}\right)^{2}}-\sum_{i=1}^{N} f_{i} \frac{\partial}{\partial x_{i}} .
$$

We show now that similar to Calogero model, its extension (3) also represents a quantum integrable system with required number of conserved quantities. The Lax operator of this system is a $N \times N$ matrix with elements

$$
L_{j k}=i\left(-\frac{\partial}{\partial x_{j}}+\delta f_{j}\right) \delta_{j k}+i \tilde{\nu} \frac{\left(1-\delta_{j k}\right)}{x_{j}-x_{k}},
$$

which reproduces the $L$ operator of Calogero model at $\delta \rightarrow 0$ limit. The complementary Lax operator $M$ on the other hand has the same form as in Calogero model [16], but with its parameter $\nu$ replaced by $\tilde{\nu}$. This pair of operators satisfy the Lax equation $\left[H, L^{ \pm}\right]=\left[L^{ \pm}, M\right] \pm \omega L^{ \pm}$, where $L^{ \pm}=L \pm \omega Q$ with $Q_{j k}=i x_{j} \delta_{j k}$. This Lax equation leads to the set of conserved quantities $I_{m}=\sum_{j k}\left(\left(L^{+} L^{-}\right)^{m}\right)_{j k}, m=1,2,3, \ldots$, ensuring the integrability of the system. Note that the Hamiltonian (3) of the extended model coincides with the conserved quantity $\frac{I_{1}}{2}+E_{g r}$.

We would like to point out here that the proposed Hamiltonian (3) lacks hermiticity property when $\delta$ and $g$ are real parameters. However, we can easily construct another variant of this model by adding a momentum dependent interaction like $H_{p}^{\prime}=\frac{\tilde{\delta}}{2} \sum_{j=1}^{N}\left(f_{j} p_{j}+p_{j} f_{j}\right)$, with $p_{j}=-i \frac{\partial}{\partial x_{j}}$, to the original Calogero model. This clearly leads to a hermitian Hamiltonian of the form

$$
H=-\frac{1}{2} \sum_{i=1}^{N} \frac{\partial^{2}}{\partial x_{i}^{2}}+\frac{\omega^{2}}{2} \sum_{i=1}^{N} x_{i}^{2}+\frac{\tilde{g}}{2} \sum_{i \neq j} \frac{1}{\left(x_{i}-x_{j}\right)^{2}}-i \frac{\tilde{\delta}}{2} \sum_{j=1}^{N}\left(f_{j} \frac{\partial}{\partial x_{j}}+\frac{\partial}{\partial x_{j}} f_{j}\right),
$$

where $\tilde{g}$ and $\tilde{\delta}$ are real parameters. It is remarkable that (16) can be expressed again in the form (3), where the coupling constants of the model are given by

$$
g=\tilde{g}+i \tilde{\delta}, \quad \delta=-i \tilde{\delta} .
$$

Therefore all the procedures followed above for solving the eigenvalue problem of (3) go through exactly in the same way for the hermitian variant (16). This however leads to some subtleties. In particular, from (7) we see that the 'exchange statistics' parameter $\nu$ becomes complex valued in this case and its real and imaginary parts can be expressed through the coupling constants as

$$
\begin{aligned}
\nu & =\nu_{R}+i \nu_{I}, \\
\nu_{R} & =\frac{1}{2}\left(1 \pm \sqrt{1+4\left(\tilde{g}-\tilde{\delta}^{2}\right)}\right), \quad \nu_{I}=-\tilde{\delta},
\end{aligned}
$$

for the choice $\tilde{g} \geq \tilde{\delta}^{2}-\frac{1}{4}$. Combining relations (17) and (18) we get $\tilde{\nu}=\nu-\delta=\nu_{R}$, i.e. the exclusion statistics parameter $\tilde{\nu}$ for the hermitian model (16) is given by the real part of the exchange statistics parameter. As a consequence, the energy spectrum in this case will be given by $E_{n_{1}, n_{2}, \cdots, n_{N}}=E_{g r}+\omega \sum_{i=1}^{N} n_{i}=\frac{N \omega}{2}\left[1+(N-1) \nu_{R}\right]+$ $\omega \sum_{i=1}^{N} n_{i}$. On the other hand both the real and imaginary parts of $\nu$ appear in the ground state wave function

$$
\tilde{\psi}_{g r}=\prod_{i<j}\left(x_{i}-x_{j}\right)^{-i \tilde{\delta}} \psi_{g r}^{0}
$$

where $\psi_{g r}^{0}=\prod_{i<j}\left(x_{i}-x_{j}\right)_{R}^{\nu} e^{-\frac{\omega}{2} \sum_{i=1}^{N} x_{i}^{2}}$. Note that $\nu_{R}$ depends on the redefined coupling constant $g^{\prime}=\tilde{g}-\tilde{\delta}^{2}$ exactly in the same way as $\nu$ depends on its coupling constant $g$ given by (2) in case of Calogero model. And since $\nu-\delta$ is replaced here by $\nu_{R}$, the operator $S^{-}$constructed above is also reduced to the corresponding operator for the Calogero model with redefined coupling constant. Consequently the wavefunctions for the hermitian model (16) and the Calogero model will have similar structures. Moreover, since the complex part of the wavefunctions appearing due to (19) does not affect the normalization, the normalizability of the eigenfunctions associated with the Hamiltonian (16) can be easily established by following the same argument as in the case of Calogero model. 
Thus we have established that, additional long-range interactions in Calogero model not only preserves its integrable structure leading to exact eigenvalue solutions, but also shows significantly richer exclusion statistics properties. Due to the competing effect of two different long-range interactions, one can construct a family of dual models with different sets of coupling constants, but having same exclusion statistics and same energy eigenvalues. More importantly, the non-coincidence of the exchange statistics with the exclusion statistics, a possibility in one-dimension, is explicitly realized in such models. As a consequence the dual models themselves differ in their exchange statistics. The inclusion of spin degrees of freedom and periodic boundary condition in the proposed model could be an important problem for future investigation [17].

[1] F. D. M. Haldane, Phys. Rev. Lett. 67, 937 (1991).

[2] Y. S. Wu and Y. Yu, Phys. Rev. Lett. 75, 890 (1995).

[3] X. G. Wen, Int. J. Mod. Phys. B 6, 1711 (1992).

[4] F. Calogero, J. Math. Phys. 10, 2191 (1969); 12, 419 (1971).

[5] S.B. Isakov, Mod. Phys. Lett. B8, 319 (1994); Int. Jour. Mod. Phys. A9, 2563 (1994).

[6] M.V.N. Murthy and R. Shankar, Phys. Rev. Lett. 73, 3331 (1994).

[7] A.P. Polychronakos, Les Houches Lectures (Summer 1998), hep-th/9902157.

[8] A. D. de Veigy and S. Ouvry, Phys. Rev. Lett. 72, 600 (1994); S. Ouvry, On the relation between the anyon and Calogero models, cond-mat/9907239.

[9] A. G. Shnirman, B. A. Malomed and E. Ben-Jacob, Phys. Rev. A 50, 3453 (1994).

[10] Anjan Kundu, Phys. Rev. Lett. 83, 1275 (1999).

[11] U. Aglietti, L. Griguolo, R. Jackiw, S.Y. Pi, and D. Seminara, Phys. Rev. Lett. 77, 4406 (1996).

[12] N. Gurappa and P.K. Panigrahi, Phys. Rev. B 59, R2490 (1999).

[13] K. Sogo, J. Phys. Soc. Jpn. 65, 3097 (1996).

[14] T.H. Baker and P.J. Forrester, Nucl. Phys. B 492, 682 (1997).

[15] H. Ujino, A. Nishino and M. Wadati, J. Phys. Soc. Jpn. 67, 2658 (1998).

[16] H. Ujino and M. Wadati, J. Phys. Soc. Jpn. 63, 3583 (1994).

[17] B. Basu-Mallick and Anjan Kundu, Under preparation 


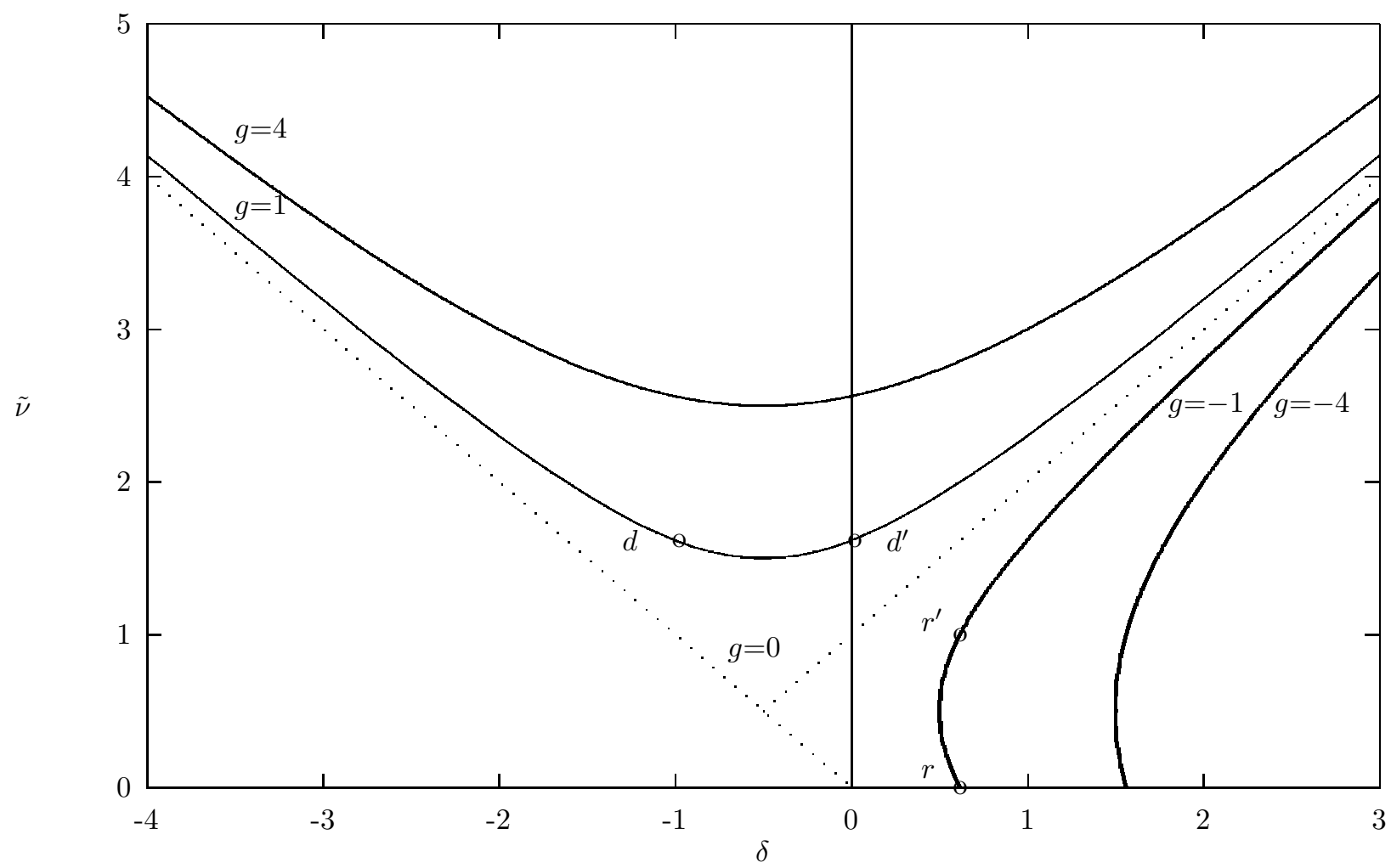

Figure 1: Plot of the exclusion statistics parameter $\tilde{\nu}$ as a function of $\delta$ for fixed values of $g= \pm 1, \pm 4$. The points $d, d^{\prime}$ correspond to dual models (14) and (1) with same $\tilde{\nu}=\frac{\sqrt{5}+1}{2}$. The points $r, r^{\prime}$ with $\delta=\frac{\sqrt{5}-1}{2}$ are related by reflection symmetry. 